\title{
STUDY OF THE FLOW FIELD AND SCALAR TRANSPORT OF INDOOR VENTILATING SPACE UNDER THE IMPACT OF COANDA VORTEX
}

\author{
Je-Ee Ho \\ Department of Mechanical Engineering, National Ilan University, Ilan, Taiwan, R.O.C., jeho@niu.edu.tw \\ Hong-Tsu Young \\ Department of Mechanical Engineering, National Taiwan University, Taipei, Taiwan, R.O.C.
}

Follow this and additional works at: https://jmstt.ntou.edu.tw/journal

Part of the Mechanical Engineering Commons

\section{Recommended Citation}

Ho, Je-Ee and Young, Hong-Tsu (2007) "STUDY OF THE FLOW FIELD AND SCALAR TRANSPORT OF INDOOR VENTILATING SPACE UNDER THE IMPACT OF COANDA VORTEX," Journal of Marine Science and Technology. Vol. 15: Iss. 2, Article 3.

DOI: $10.51400 / 2709-6998.2036$

Available at: https://jmstt.ntou.edu.tw/journal/vol15/iss2/3

This Research Article is brought to you for free and open access by Journal of Marine Science and Technology. It has been accepted for inclusion in Journal of Marine Science and Technology by an authorized editor of Journal of Marine Science and Technology. 


\title{
STUDY OF THE FLOW FIELD AND SCALAR TRANSPORT OF INDOOR VENTILATING SPACE UNDER THE IMPACT OF COANDA VORTEX
}

\author{
Je-Ee Ho* and Hong-Tsu Young**
}

Key word: flow cut, Coanda effect.

\begin{abstract}
The vortex in the flow field will isolate the space to further reduce the indoor air exchange and convective efficiency. The Coanda vortex accompanied by the inlet jet usually has the most active effect on the flow field. Both its location and scalar size which are primary determined by the strength of the injected flow and the arrangement of inlet; outlet will deeply affect the air-exchange volume, even reduce the available ventilation space. A 2-D turbulent model with SIMPLE method is set up to simulate the air motion of indoors; it can easily describe the working of Coanda vortex in the flow field. When compared with the flow pattern of Coanda vortex from Awbi (1991) and the experiment made by Kurabuchi (1990), the simulating results are proven to be agreement with those presented data and can provide available information to the indoor air motion.
\end{abstract}

\section{INTRODUCTION}

The indoor air distribution is a very complex turbulent motion which includes natural convention, force convection and mixing problem together. It significantly influences the indoor air quality and thermal comfort as well. Although relative researches on these topics have been investigated vastly in the last decade, the effect of Coanda vortex was still less mentioned before. Larry [4] built a PMV theoretical model of thermal comfort including environmental temperature and humidity parameters to define the condition of mind which expresses the satisfaction with thermal environment, Ghiaus [1] discussed the convective motion with buoyancy effect and estimated air exchange rate in an enclosure space. Shih, Chiang, and Shyu [6] referred Coanda effect to analyze indoor air quality with CFD package PHOENICS to simulate the flow and

Paper Submitted 03/27/06, Accepted 08/21/06. Author for Correspondence: Je-EeHo.E-mail: jeho@niu.edu.tw.

*Department of Mechanical Engineering, National Ilan University, Ilan, Taiwan, R.O.C.

**Department of Mechanical Engineering, National Taiwan University, Taipei, Taiwan, R.O.C. temperature distribution indoors where single air-conditioner was fixed in the room. Xing, Hotron, and Awib [7] studied the difference in air quality that was perceived by the occupants with the displacement of ventilation system. Hayashi et al. [2] examined the characteristics of contaminated air with a simplified 2-D model in which a new index IECV was introduced to show the convective efficiency in a room. In the past, the researches of tracing air technique was focused on individual experimental topics and no complete numerical analysis of Coanda effect on predicting the indoor flow field was presented. To describe the relation between the flow field and Coanda effect, the vortex behavior will be involved and investigated by changing locations of inlet; outlet and setting various strength of inlet jet. As for the parameters of humidity and temperature, they were taken as minor effects which were neglected without causing significant errors in this study.

\section{ANALYSIS}

The $\kappa-\varepsilon$ turbulent model with several conservation Eqs. such as mass continuity, momentum transport, and energy equilibrium was applied to solve the flow field and temperature distribution in this study. Through the dimensionless analysis, those governing equations based on mean value theory were discretized by the numerical skills with SIMPLE method. Meantime, an appropriate wall function was utilized to ensure the continuous property at the boundary walls.

\section{Fundamental formulations:}

Continuity Eq.:

$$
\frac{\partial \hat{u}_{j}}{\partial \hat{x}_{j}}=0
$$

Momentum Eq.:

$$
\frac{\partial \hat{u}_{i} \partial \hat{u}_{j}}{\partial \hat{x}_{i}}=\frac{-\partial P}{\partial \hat{x}_{i}}+\frac{\partial}{\partial \hat{x}_{j}}\left\{\hat{v}_{e f f}\left(\frac{\partial \hat{u}_{i}}{\partial \hat{x}_{j}}+\frac{\partial \hat{u}_{j}}{\partial \hat{x}_{i}}\right)\right\}
$$


$\kappa \mathrm{Eq} .:$

$\frac{\partial k \hat{u}_{j}}{\partial \hat{x}_{j}}=\frac{\partial}{\partial \hat{x}_{j}}\left[P_{k} \frac{\partial \hat{k}}{\partial \hat{x}_{j}}\right]+\hat{v}_{t} S-\hat{\varepsilon}$

$\varepsilon \mathrm{Eq}:$ :

$\frac{\partial \hat{\varepsilon} \hat{u}_{j}}{\partial \hat{x}_{j}}=\frac{\partial}{\partial \hat{x}_{j}}\left[P_{\varepsilon} \frac{\partial \hat{\varepsilon}}{\partial \hat{x}_{j}}\right]+\frac{\hat{\varepsilon}}{\hat{k}}\left(c_{1} \hat{v}_{t}-c_{2} \widehat{\varepsilon}\right)$

temperature Eq.:

$$
\frac{\partial \hat{\theta} \hat{u}_{j}}{\partial \hat{x}_{j}}=\frac{\partial}{\partial \hat{x}_{j}}\left[\alpha_{e f f} \frac{\partial \hat{\theta}}{\partial \hat{x}_{j}}\right]
$$

boundary condition:

(1) boundary wall:

$$
\begin{aligned}
& \hat{u}_{i}=0 \\
& \frac{\partial \hat{k}}{\partial x_{i}}=0, \frac{\partial \hat{\varepsilon}}{\partial x_{i}}=0, \frac{\partial \hat{p}}{\partial x_{i}}=0, \hat{\theta}=0.8
\end{aligned}
$$

(2) inlet, outlet:

$$
\hat{u}=U_{0}
$$

$\hat{k}=0.005 \hat{u}^{2}, \hat{\varepsilon}=0.00032 \hat{u}^{3} / L_{0}[$ Kurabuchi, 1990]

$\hat{\theta}=0.5$ (inlet), $\frac{\partial \hat{\theta}}{\partial x}=0$ (outlet)

$\hat{P}=\hat{P}_{\text {ambinet }}($ outlet $)$

\section{Numerical method}

As refer to the reference [5], a two dimensional discretization equation can expressed as the following generalized equation by the SIMPLE method with power law scheme.

$$
a_{p} \hat{\phi}_{p}=\Sigma a_{I} \hat{\phi}_{I}+b
$$

where $a_{p}=\Sigma a_{I}$

$$
\begin{aligned}
& b= \begin{cases}\left(P_{p}-P_{i}\right) \delta x_{j} & \text { for moment equation } \\
S \delta x_{i} \delta x_{j} & \text { for other equations }\end{cases} \\
& a_{I}=D_{i}\left\{\mathrm{~A}\left(\left|P_{i}\right|\right)+\left[-P_{i}, 0\right]\right\}
\end{aligned}
$$

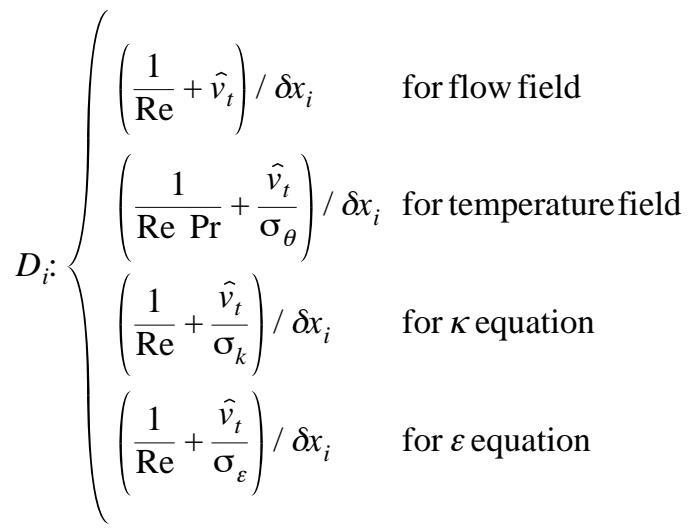

$P_{i}=D_{i} \times \hat{u}_{i}$

power law scheme:

$A\left(\left|P_{i}\right|\right)=\left\lfloor 0,1-0.1\left|P_{i}\right|^{5}\right\rfloor$

$\mathrm{i}=\mathrm{e}, \mathrm{w}, \mathrm{n}, \mathrm{s}$ corresponds to the index $\mathrm{I}=\mathrm{E}, \mathrm{W}, \mathrm{N}, \mathrm{S}$ (Figure 1)

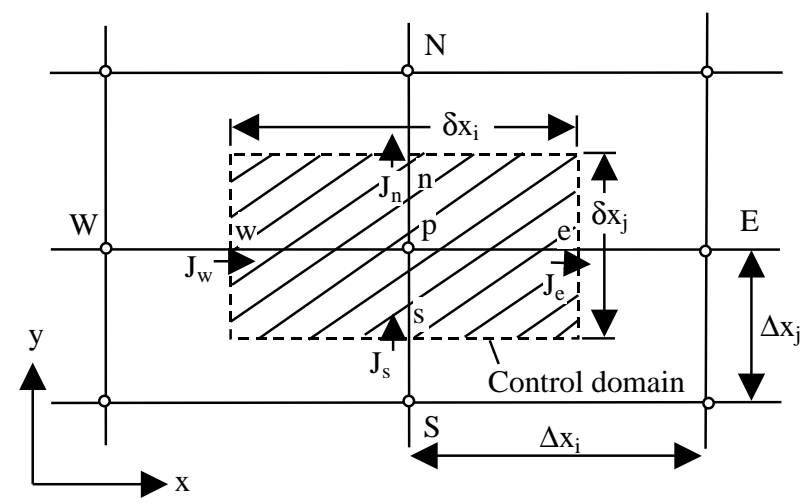

Fig. 1. Grids arrangement in 2-D calculating domain.

\section{Numerical procedure}

The discrete form of Eqs. (1) (5) with boundary conditions were obtained by SIMPLE method (Patankar [5]) and the final form has been simplified as Eq. (6). A grid of $20 \times 30$ nodal points ensured independence of the solution on the grid. The nodal points in both $\mathrm{x}$ and $\mathrm{y}$ direction were uniformly distribution, but the step size $\Delta x=0.05, \Delta y=0.033$ were set up to provide a square calculation domain. For the grids test, the nodal points with $20 \times 30,30 \times 45,40 \times 60$ were selected to perform the simulation program. The results showed that the maximum relative errors in the corresponding physical quantities were not more than $3 \%$. It implied the adopt of grids $20 \times 30$ was appropriate to be used in the 
simulating program without causing the significant errors. The key steps for solving this problem are as follows.

(1) Set the locations of inlet and outlet.

(2) Given the flow velocity of inlet jet.

(3) All the discretization equations are solved with boundary conditions by using the successive over relaxation method with a relaxation factor 1.2.

(4) If the relative errors in all the corresponding physical quantities are less than $0.5 \%$, another velocity of inlet jet is set and repeat the steps (3) (4). Otherwise, the step c will keep running.

(5) Various locations of inlet and outlet are arranged and repeat the step (2) (4).

\section{RESULTS AND DISCUSSION}

Coanda effect is usually found in air-ventilating system indoors. It can be described as the vortex accompanied with inlet jet makes the flow spray out to strength or weak the convective effect in the closed space. The flow field under the effect of Coanda vortex can be classified into three different zones as shown in Figure 2.

Vorticity zone: The region with a low pressure flow usually appears around the inlet jet. It also exhibits an isolated region with worse air ventilation efficiency and high contaminant concentration.

Inertial zone: The flow is drawn vertically by vortex and travels along the wall. Its direction will be not changed till the effect of gravity or the lower pressure near the exit. In this region, it will promote higher convection effect and air exchange rate.

Outlet zone: Due to lower pressure at outlet, the higher flow velocity will be observed at the exit.

When inlet and outlet are arranged as Figure 3. indicates, both small Coanda vortexes coupled with the colder jet $\theta=0.5, \mathrm{u}=0.1$ will draw the flow upwards; downwards and then spray it around the whole area.

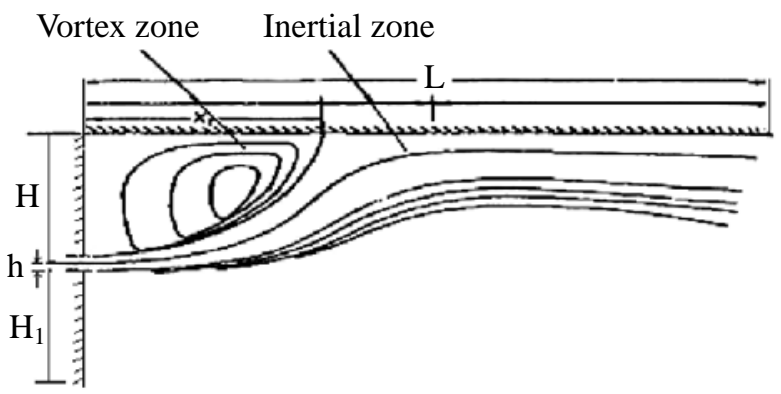

Fig. 2. The sketch of Coanda Effect induced by inlet jet (refer to reference [7]).
The area covered by the inertial contour ABCDA is defined as the available air exchange region where is almost $75 \%$ total area. The wider spraying angle is; the better of air mixing exchange and convection effect present.

The Figure 4 demonstrates the distribution of nondimensional temperature. The temperature in the region bounded by isothermal line $\theta=0.7$ nearly keeps constant along the flow path, but varies significantly in the normal to flow direction. It implies that a better convective effect will be observed along streamlines and the conduction will become important in its normal direction. When compare the convective region bounded by $\theta=0.7$, it nearly collides with the available air exchange region covered by ABCDA in Figure 3. As for the locations of the Coanda vortex, the constant temperature is kept about 0.8 around there where a poor connective efficiency will be predicted.

For the in jet with the velocity 1.0 in Figure 5, the

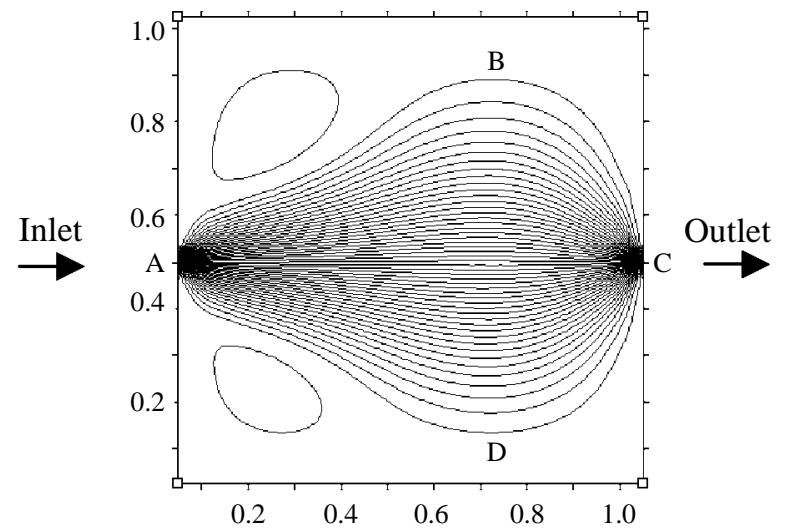

Fig. 3. Stream line distribution for $\mathbf{U}_{\text {inlet }}=0.1$ and the location of inlet and outlet are arranged on the center of opposite side.

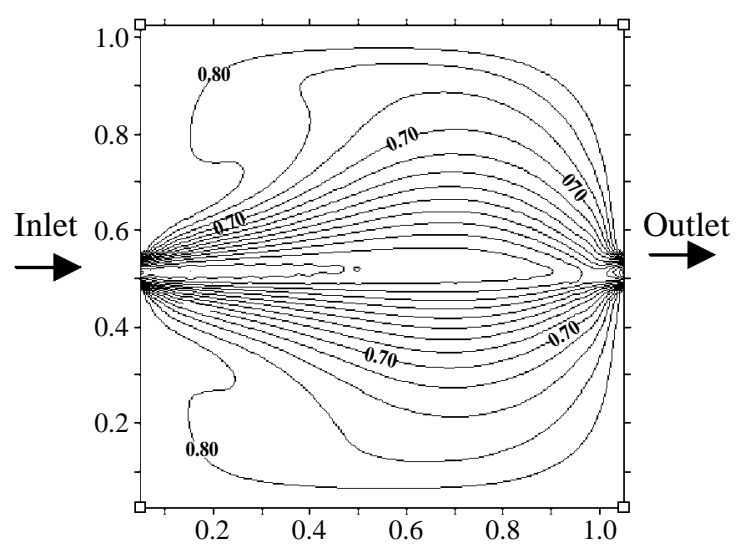

Fig. 4. Temperature distribution for $\mathbf{U}_{\text {inlet }}=0.1$ while the location of inlet and outlet are arranged on the center of opposite side. 
size of vortexes will grow gradually during the process as they are induced away from the inlet by strong inlet flow. It will make the spraying out of air flow decreased, even be vanished and the available veniality area will be further restricted to the region nearby the path $\mathrm{G} \rightarrow$ $\mathrm{H}$ where only takes about $20 \%$ total area.

The discussion of temperature distribution is shown in Figure 6. The effective convection is only existed nearby the path $\mathrm{G} \rightarrow \mathrm{H}$ where is bounded by $\theta=0.7$ and it is approximate consistent with the available ventilation region in Figure 5. However, the worst air exchange regions with temperature about $0.7 \sim 0.8$ have occupied $80 \%$ total area where is just collided with the space Coanda vortexes cover.

When the slower inlet velocity 0.1 is applied with the display of inlet and outlet as in Figure 7, the vortex doesn't appear in the flow field. It indicates the inlet flow can spray out well except at the left-down and right-up corners where the air motion is very slow.

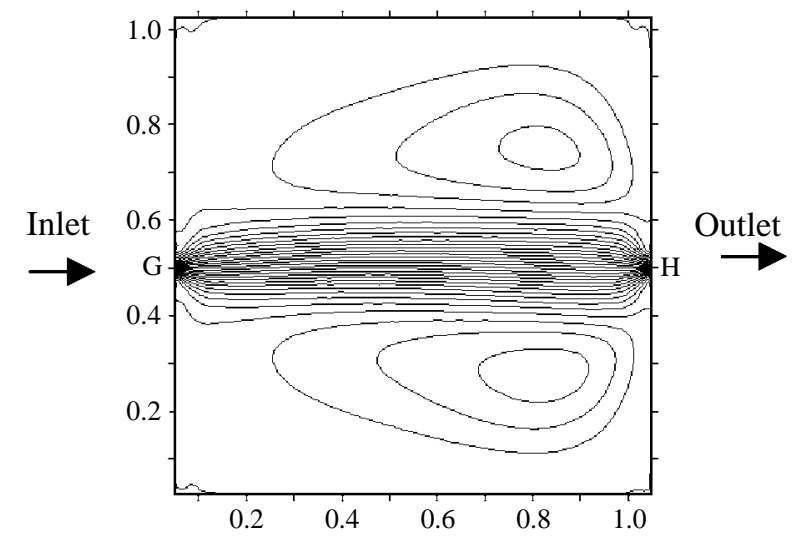

Fig. 5. Stream line distribution for $\mathbf{U}_{\text {inlet }}=1.0$ while the location of inlet and outlet are arranged on the center of opposite side.

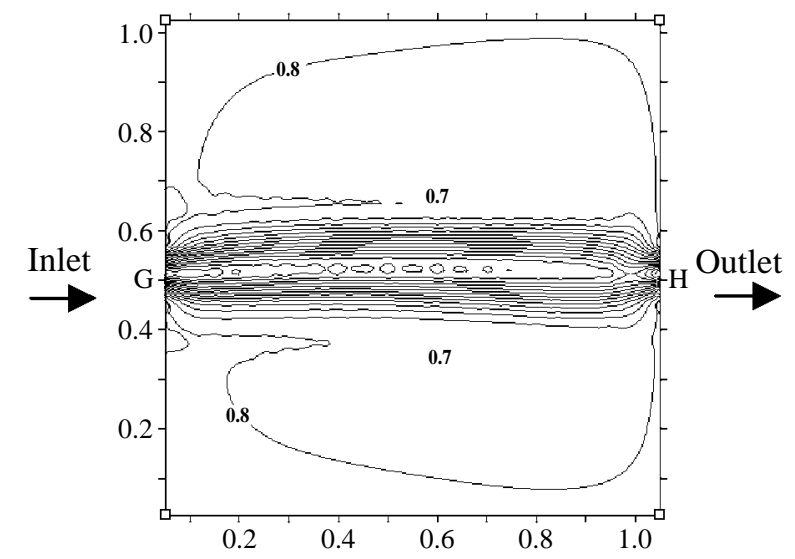

Fig. 6. Temperature distribution for $U_{\text {inlet }}=1.0$ while the location of inlet and outlet are arranged on the center corner of opposite side.
From this arrangement, most of the space will be available ventilation region, especially at the place where is along the directed path from inlet to outlet.

With the higher inlet flow velocity 1.0, a larger vortex distorting the flow path is observed around the left-down corner in Figure 8. It tells that a worse air exchange will be presented at there and available ventilation area along the inlet to outlet will be much decreased to about $20 \%$ total area.

As a small inlet flow velocity 0.1 is injected into the space with the display of inlet and outlet in Figure 9, the best air exchange with the smooth of spraying out flow is reached. In this case, not any vortex appears except both small idle regions with slow air motion are observed at the opposite wall and near the inlet at the left wall. From the streamline distribution shows, available ventilation region covers most of the space and takes about $75 \%$ total area.

If the velocity of inlet flow is increased to 1.0 , the

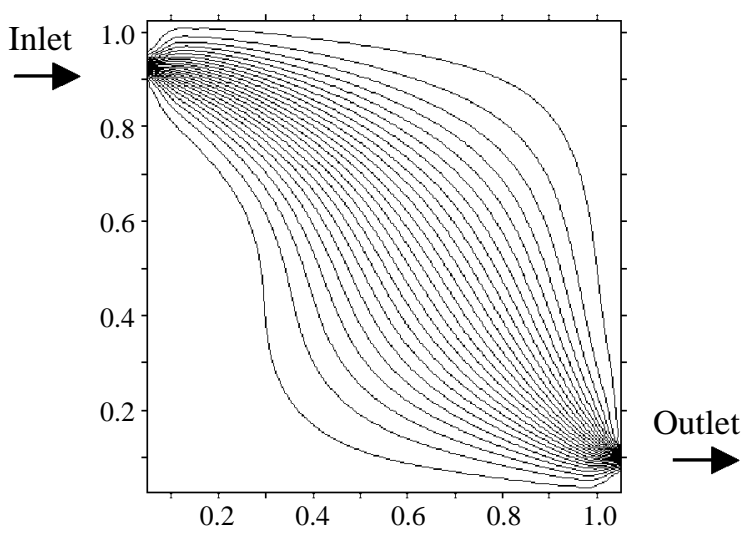

Fig. 7. Stream line distribution for $U_{\text {inlet }}=1.0$ while the location of inlet and outlet are arranged on the different corner of opposite side.

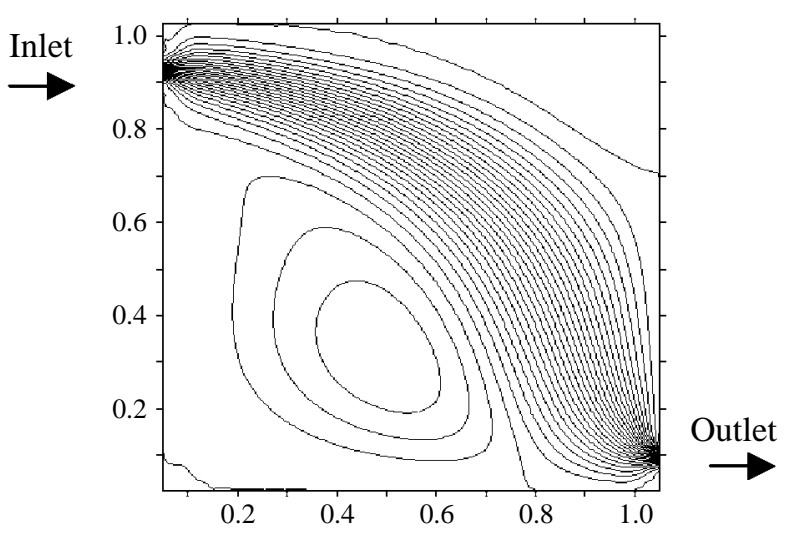

Fig. 8. Stream line distribution for $U_{\text {inlet }}=1.0$ while the location of inlet and outlet are arranged on the different corner of opposite side. 
idle region extends from opposite wall and a Coanda vortex also appears at the central portion of left side where a poor convective effect will be predicted. From the demonstration in Figure 10, not only the flow path is

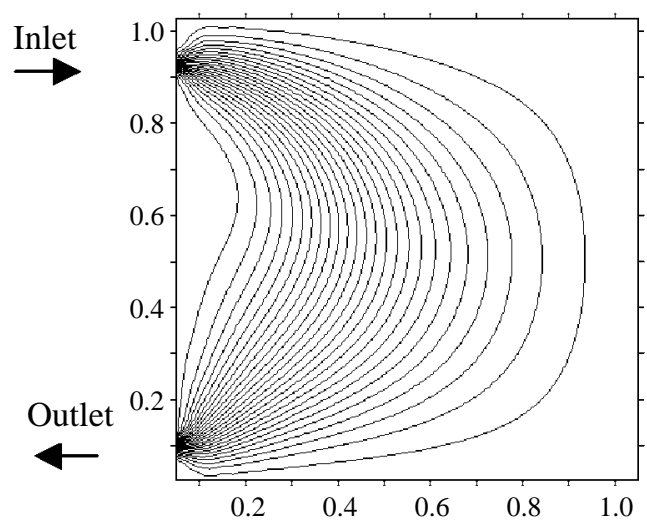

Fig. 9. Stream line distribution for $U_{\text {inlet }}=0.1$ while the location of inlet and outlet are arranged on the different corner of same side.

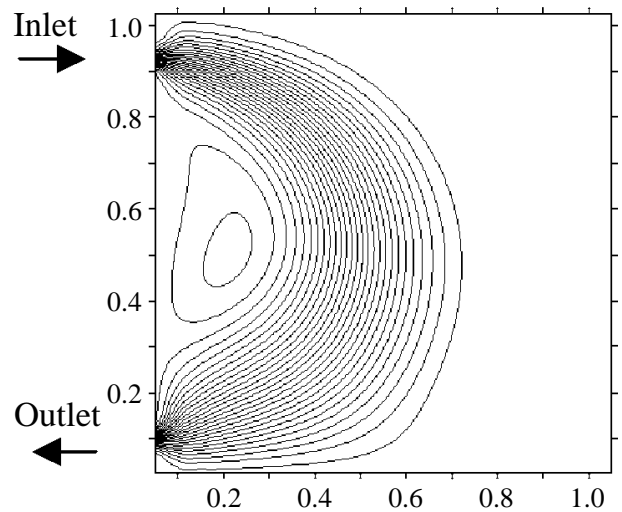

Fig. 10. Stream line distribution for $U_{\text {inlet }}=1.0$ while the location of inlet and outlet are arranged on the different corner with the same side.

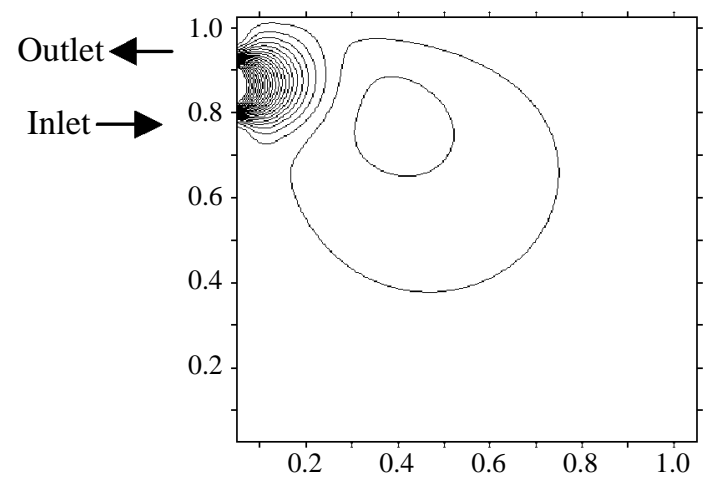

Fig. 11. Stream line distribution for flow cut. significantly distorted by Coanda vortex, but it will further reduce the available air exchange area to be about $30 \%$ total area.

Another worthy discussion is the phenomena of flow cut. Locating the inlet closer to the outlet and set the outlet near the wall as shown in Figure 11, we find that the channel of outlet flow will be further reduced by the wall and inlet. It implies that the motion of out flow will be much faster than the inlet flow moves by following the continuity theorem, which just likes a strong sucking effect at the outlet. Once the inlet flow is injected into the room, the air will be sucked back to the outlet immediately and the spraying out of air seems impossible. That will produce a flow cut in space and the air exchange region will be further reduced to the minimum which is about $5 \%$ total areas.

When compared with Awbi's experiment in Figure 2 , the prediction of the location of Coanda vortex and indoor flow path from Figure 3 seem to be kept well with the experiment data. Another experiment of tracing passive makers in the flow field was made by Kurabuchi et al. [3]. Its result in Figure 12 showed that two vortexes appeared near the inlet and a larger circulation existed at the left side in space. That is agreement with the simulating display in Figure 13. However, more particles were observed to gather at the left-down corner in Figure 12 where the slow air motion would be predicted. That was also similar to the simulating behavior displayed in the Figure 13 .

\section{CONCLUSIONS}

1. The available ventilation area nearly collides with the effective convective region where is bounded by isothermal $\theta=0.7$.

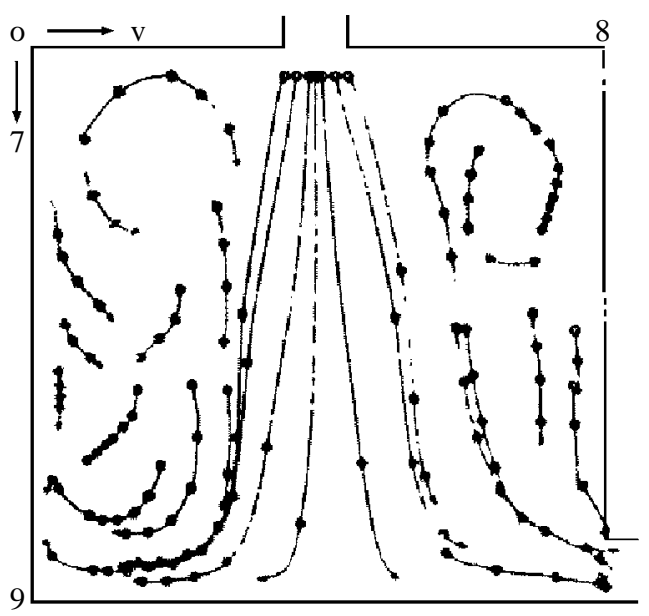

Fig. 12. Trajectories of Passive Markers Based on Experimental Data made by Kurabuchi. 


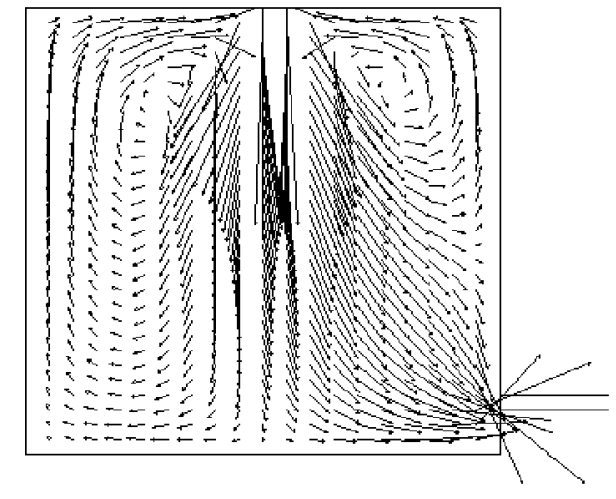

Fig. 13. The simulating of flow distribution in vector field.

2. The temperature almost remains constant along flow streamline while the temperature gradient significantly varies in normal to streamline. That means the convective effect seems obvious along jet flow path and the conduction terms will be dominated in the normal direction of the flow path.

3. At the lower inlet velocity case, the smaller Coanda effect tends to spray out the jet to increase the air exchange region and convective effect.

4. In contrary to conclusion 3 , higher inlet velocity increases the size of vortex that will form large isolated regions in the space and low down the air exchange efficiency.

5 . When the inlet and outlet diffusers are located very close, the flow cut phenomenon will cause the worst convective efficiency.

6. As slow inlet jet is applied, the maximum available air exchange area and convective effect will be produced by arranging the inlet and outlet as the same side with different corner. But for high speed jet, no obvious difference in efficiency is observed except the poor performance of flow cut.

\section{NOMENCLATURE}

a coefficient in the discretization equation

$S$ general source term

$\delta_{i} \quad$ i-direction distance between two adjacent grid points

$\delta_{\mathrm{j}} \quad \mathrm{j}$-direction distance between two adjacent grid points

$\mathrm{x}_{\mathrm{i}} \quad$ coordinate in $\mathrm{i}$ component $(\mathrm{m})$

$x_{j} \quad$ coordinate in $j$ component $(m)$

$\mathrm{u}_{\mathrm{j}} \quad$ local velocity in $\mathrm{j}$ component $(\mathrm{m} / \mathrm{s})$

$P \quad$ local air pressure $(\mathrm{Pa})$

$\kappa \quad$ turbulence energy $(\mathrm{m} / \mathrm{s})^{2}$

$\varepsilon \quad$ dissipation rate of turbulence energy $\left(\mathrm{m} / \mathrm{s}^{2}\right)$

$v \quad$ instantaneous viscosity of $\operatorname{air}\left(\mathrm{m}^{2} / \mathrm{s}\right)$

$\alpha$ thermal diffusivity of air $\left(\mathrm{m}^{2} / \mathrm{s}\right)$ $\mu \quad$ dynamic viscosity of air $(\mathrm{kg} / \mathrm{ms})$

$\mathrm{L}_{0}$ is the width of the inlet opening $(\mathrm{m})$

$\mathrm{T}_{\omega} \quad$ wall temperature

$\mathrm{T}_{\infty}$ ambient temperature

$\mathrm{U}_{0}$ is the inlet air velocity $(\mathrm{m} / \mathrm{s})$

$\phi$ general dependent variable

$\mathrm{P} \quad$ central grid point under consideration

$v_{t}=C_{D} \frac{\kappa^{2}}{\varepsilon}$ eddy viscosity

$\hat{x}_{j}=x_{j} / L_{0}$ dimensionless coordinate in $\mathrm{j}$ component

$\hat{u}_{j}=u_{j} / U_{0}$ dimensionless velocity of air

$\theta=\frac{T-T \infty}{T w-T \infty}$ dimensionless local temperature

$\hat{\rho}=\rho / \rho_{0}$ dimensionless density of air

$\hat{p}=p / \rho_{0} U_{0}^{2}$ dimensionless local pressure of air

$\hat{k}=k / U_{0}^{2}$ dimensionless turbulence energy

$\hat{\varepsilon}=\varepsilon L_{0} / U_{0}^{3}$ dimensionless dissipation rate of turbulent

energy

$\hat{v}_{t}=\frac{v_{t}}{U_{0} L_{0}}$ dimensionless eddy viscosity

$v_{e f f}=\frac{1}{R_{e}}+\hat{v}_{t}$ effective eddy viscosity

$\alpha_{e f f}=\frac{1}{R_{e} S_{c}}+\frac{\hat{v}_{t}}{\sigma_{c}}$ effective thermal diffusivity

$P_{k}=\frac{1}{R_{e}}+\frac{\hat{v}_{t}}{\sigma_{k}}$ effective turbulence energy diffusivity

$P_{\varepsilon}=\frac{1}{R_{e}}+\frac{\hat{v}_{t}}{\sigma_{\varepsilon}}$ effective dissipation rate of turbulent

$\operatorname{Re}=L_{0} U_{0} / v$ Renold number

$P_{i}=$ Pelect number

$p_{r}=\frac{v}{\alpha}$ Prantal number

$S=\left(\frac{\partial \hat{u}_{i}}{\partial \hat{x}_{j}}+\frac{\partial \hat{u}_{j}}{\partial \hat{x}_{i}}\right) \frac{\partial \hat{u}_{i}}{\partial \hat{x}_{j}}$

Empirical constants: $\mathrm{c}_{\mathrm{D}}=0.09, \mathrm{c}_{1}=1.44, \mathrm{c}_{2}=1.92$, $\mathrm{c}_{3}=1.0, \sigma_{k}=1.0, \sigma_{\varepsilon}=1.3, \sigma_{\theta}=0.9$,

\section{REFERENCES}

1. Ghiaus, C.M. and Ghiaus, A.G., "Evaluation of the Indoor Temperature Field using a Given Air Velocity 
Distribution," Building and Environment, Vol. 34, pp. 671-679 (1999).

2. Hayashi, T., Ishizu, Y., Kato, S., and Murakami, S., "CFD Analysis on Characteristics of Contaminated Indoor Air Ventilation and its Application in the Evaluation of the Effects of Contaminant in Halation by a Human Occupant," Building and Environment, Vol. 37, pp. 219-230 (2002).

3. Kurabuchi, T., Fang, J.B., and Grot, R.A., A Numerical Method for Calculating Indoor Airflows Using a Turbulence Model (NIST Report R89-4211), National Institution of Technology and Standards, USA (1990).
4. Larry, P.E, "Comfort and Humidity," ASHRAE Journal, Vol. 40, No. 8, pp. 35-41 (1998).

5. Patankar, S.V., Numerical Heat Transfer and Fluid Flow, Hemisphere, Washington D.C. (1980).

6. Shih, Y.C., Chiang, H., and Shyu, R.T., "The Impact of the Coanda Effect on the Indoor Air Motion Generated by a Window-Type Air-Conditioner," The Conference of Roommvent, pp. 9-12 (2000).

7. Xing, H., Hatton, A., and Awbi, H.B., "A Study of Air Quality in the Breathing Zone in a Room with Displacement Ventilation," Building and Environment, Vol. 36, pp. 809-820 (2001). 